\title{
Editorial
}

\section{Topological Methods in Analysis}

\author{
Salvador Hernández, ${ }^{1}$ Lawrence Narici, ${ }^{2}$ and Javier Trigos-Arrieta ${ }^{3}$ \\ ${ }^{1}$ Departamento de Matemáticas, Universitat Jaume I, Campus de Riu Sec, 12071 Castellón, Spain \\ ${ }^{2}$ Department of Mathematics, St. Johns University, Queens, New York City, NY 11439, USA \\ ${ }^{3}$ Department of Mathematics, California State University, Bakersfield, Bakersfield, CA 93311, USA
}

Correspondence should be addressed to Salvador Hernández; hernande@mat.uji.es

Received 11 November 2013; Accepted 11 November 2013

Copyright (C) 2013 Salvador Hernández et al. This is an open access article distributed under the Creative Commons Attribution License, which permits unrestricted use, distribution, and reproduction in any medium, provided the original work is properly cited.

Topological methods have played a seminal role in functional analysis since its birth in the early twentieth century. The Baire category theorem, for example, is the bedrock on which rest such basic principles of functional analysis as the open mapping theorem and the principle of uniform boundedness. Initial (weak) topologies, compactness, and the Tikhonov theorem drive such classical results of duality theory as the Banach-Alaoglu and Krein-Milman theorems. Topological methods also play a crucial role in Banach algebra theory (Gelfand topology), harmonic analysis (locally compact groups and function spaces), differential equations (fixed point theorems and Ascoli-Arzela theorem), and nonlinear analysis (fixed point existence theorems and topological degree theory) to mention just a few.

The lofty goal of this special issue is to present some new outstanding contributions in this research area. They include results on fixed point theorems, metric spaces, $C^{*}$-algebras, modular spaces, algebraic hypersurfaces, and topological groups. Our hope is that this wide spectrum of topics will be of interest to many researchers in the area.

\section{Acknowledgment}

To the referees whose time and expertise have made this volume possible we say: Thank you! Without your generous toil, it would not exist.

Salvador Hernández Lawrence Narici Javier Trigos-Arrieta 


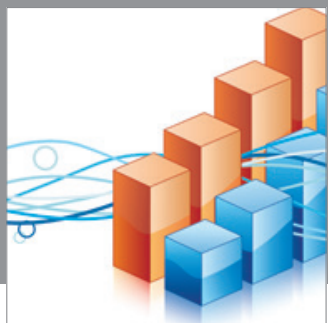

Advances in

Operations Research

mansans

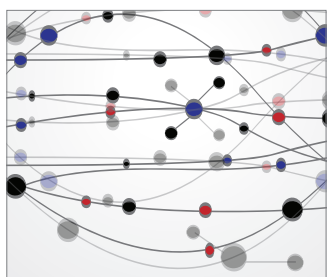

The Scientific World Journal
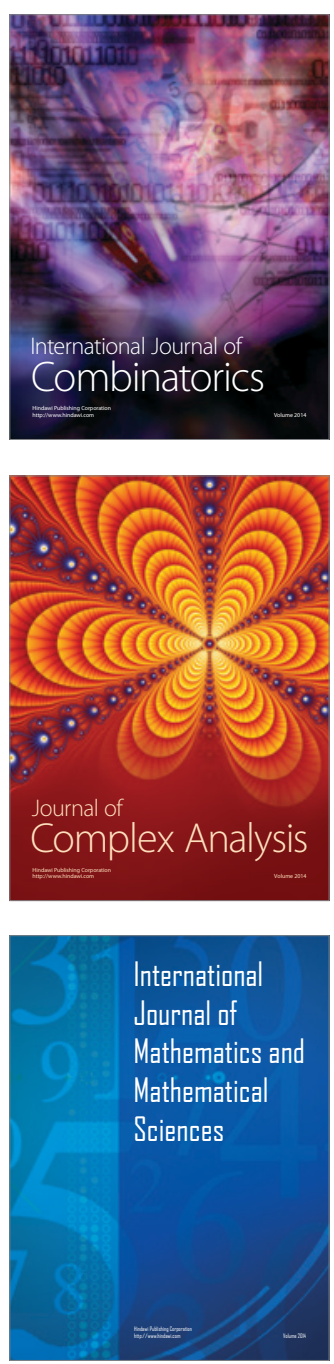
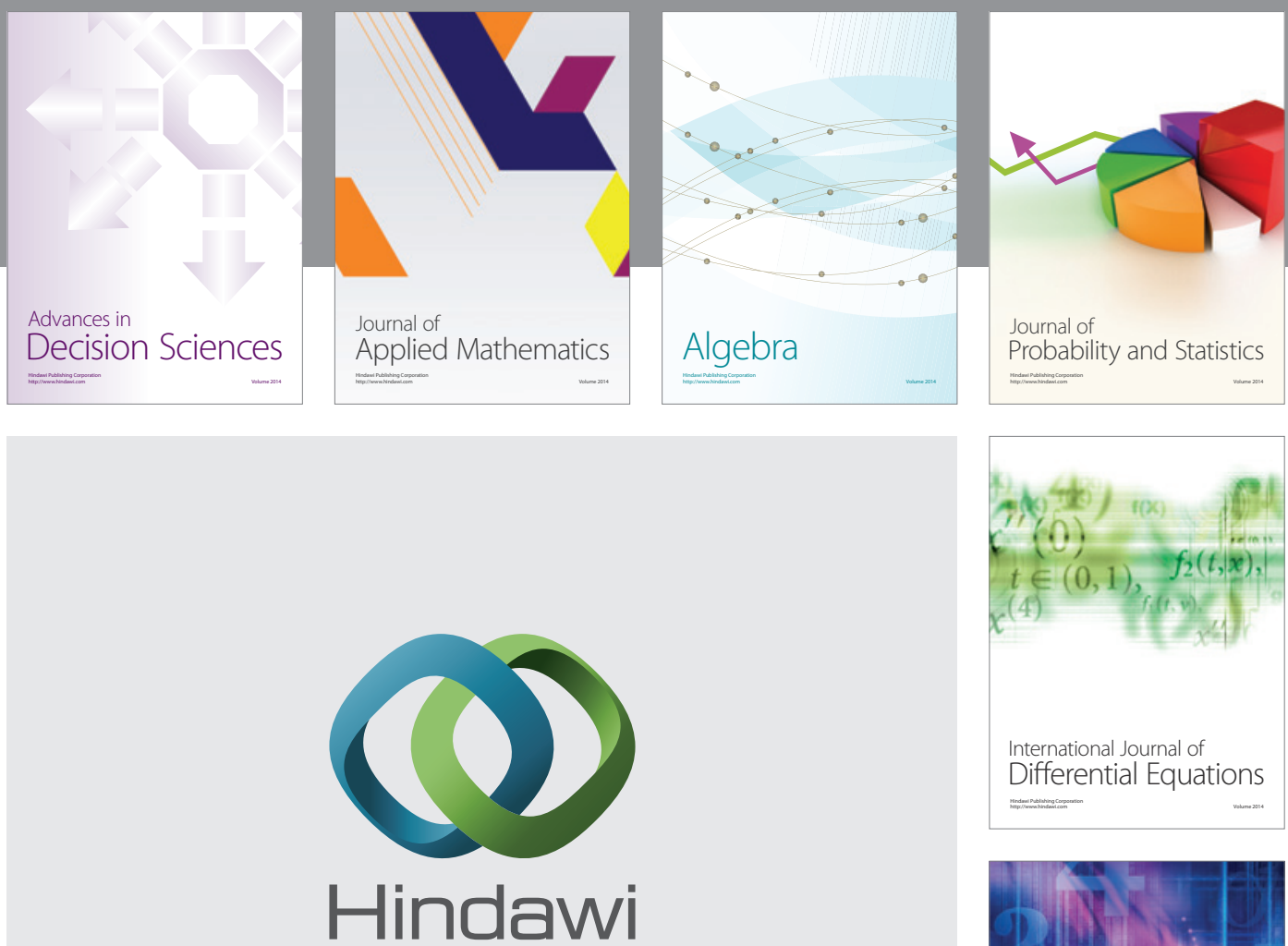

Submit your manuscripts at http://www.hindawi.com
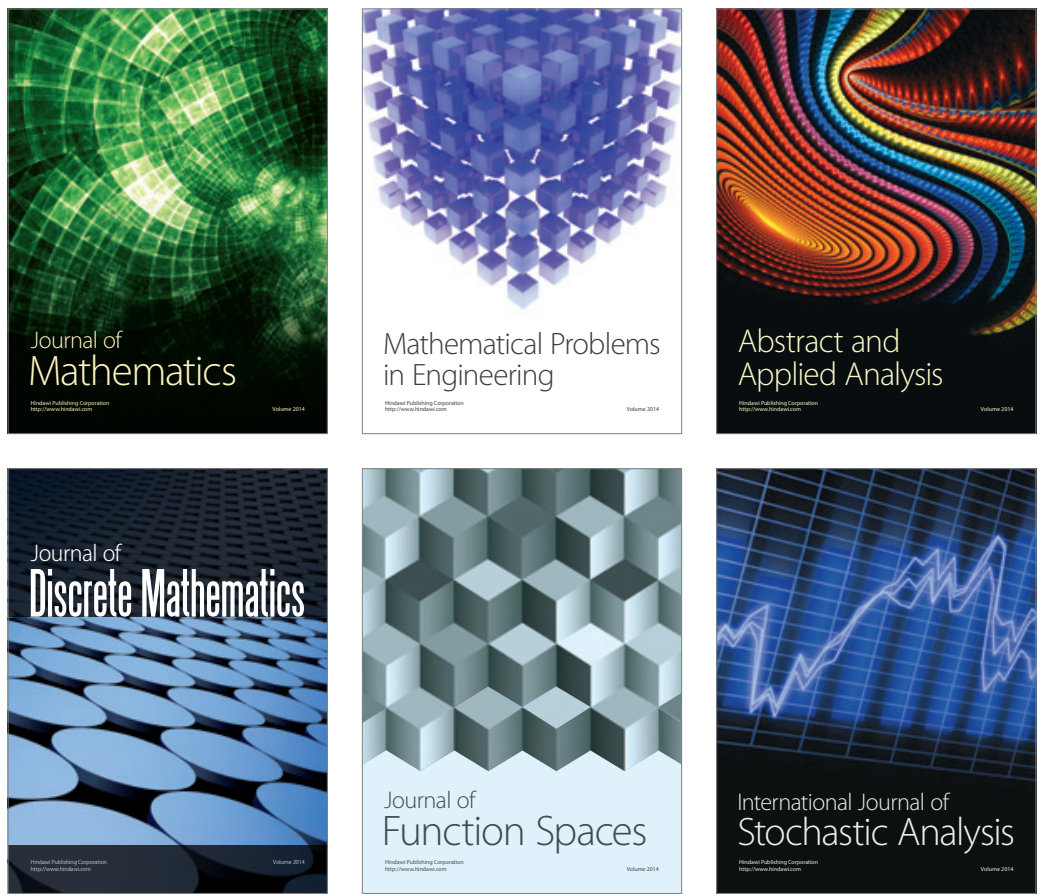

Journal of

Function Spaces

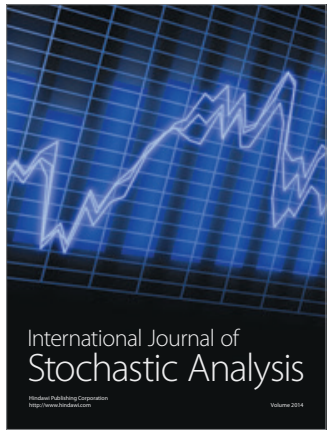

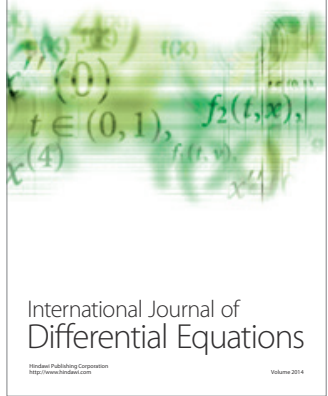
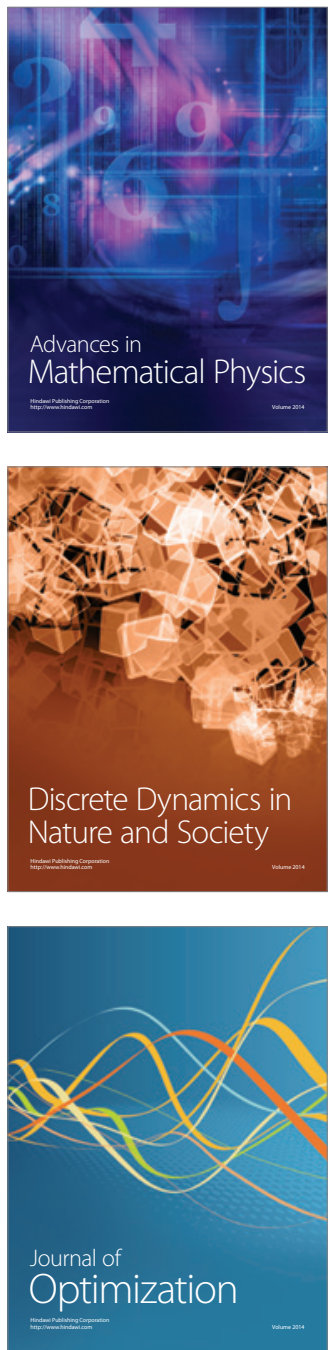